NUB 3054/92

\title{
Compact Lattice QED and the Coulomb Potential
}

\author{
Y.N. Srivastava, A. Widom and M.H. Friedman \\ Physics Department, Northeastern University \\ Boston, Massachusetts, U.S.A. \\ and \\ Physics Department \& INFN., University of Perugia \\ Perugia, Italy \\ and \\ O. Panella \\ Centre de Physique Theorique, CNRS Luminy \\ Marseille, France
}

ABSTRACT

The potential energy of a static charge distribution on a lattice is rigorously computed in the standard compact quantum electrodynamic model. The method used follows closely that of Weyl for ordinary quantum electrodynamics in continuous space-time. The potential energy of the static charge distribution is independent of temperature and can be calculated from the lattice version of Poisson's equation. It is the usual Coulomb potential. 
The notion of approximating gauge field theories in continuous space-time by discrete lattice gauge models grew (in part) with the desire to compute numerically the consequences of quantum gauge theories ${ }^{(1-5)}$. This might be accomplished without recourse to perturbative methods. However, some features of the discrete lattice version of QED theory appear more difficult than in the continuous space-time theory. Such appears to be the case for the force law between charges in compact lattice quantum electrodynamics ${ }^{(6-13)}$.

For quantum electrodynamics in continuous space-time, the law of force between two static charges is that of Coulomb, while the law of force between two steady state current distributions follows from Ampere's law. For lattice compact quantum electrodynamics, the Ampere law can become somewhat difficult to compute due to the inherent nonlinear form of the field equations. On the other hand, the lattice compact quantum electrodynamic analog of the Coulomb law can be directly computed. This lattice Coulomb law calculation is the purpose of the work which follows.

The compact quantum electrodynamic lattice model (to be discussed) is here described in the canonical Hamiltonian form. The cubic lattice of the theory (three space dimensions) has vertices located at positions $\{\mathbf{x}\}$. The components of a vector field at a vertex live on the links $\{L\}$ connecting neighboring vertices. In addition to the links connecting neighboring vertices, one must consider the plaquettes $\{P\}$. These are the square areas bounded by four links. An alternative view employs the dual lattice with vertices located at $\left\{\mathbf{x}^{*}\right\}$ which are the center points of the cubes on the original lattice. The links on the dual lattice are in one to one correspondence to the plaquettes on the original lattice. Each link on the dual lattice punctures a unique plaquette area on the original lattice.

The electric field components live on the links of the original lattice. The magetic field components live on the links of the dual lattice, or equivalently on the plaquettes of the original lattice as the Faraday law magnetic flux through the plaquette area.

Perhaps the most simple manner to describe QED on a lattice is to employ a "quantum circuit array" viewpoint. (We use units where $\hbar=1$ and $c=1$.) The (temporal gauge) relation between the electric field and the vector potential,

$$
\mathbf{E}(\mathbf{x})=-\dot{\mathbf{A}}(\mathbf{x})
$$

on the lattice appears as an expression for the voltage across a link

$$
V_{L}=-\dot{\phi}_{L}
$$

The energy stored in the electric field is defined by assigning (to each link) a capcitance C, so that the energy becomes

$$
K=(1 / 2) C \sum_{L} V_{L}^{2}
$$

If we sum the voltages around the links (which form the boundary of a plaquette), then the lattice version of Faraday's law is that we must obtain the rate of change of the magnetic flux through the plaquette. Formally, Faraday's law on the lattice arises as a consequence of the definition of magetic flux $\Phi_{P}$ through a plaquette. It is

$$
\exp \left(i q \Phi_{P}\right)=\prod_{L \in \partial P} \exp \left(i q \eta_{L} \phi_{L}\right)
$$

where $q$ is the "charge" unit of the theory, $\partial P$ is the boundary of the plaquette, and the coefficients $\eta_{L}= \pm 1$ dictate the orientaion of the plaqutte area. That we are discussing compact QED on the lattice now makes an appearance since the inductance $\Lambda$ (assigned to each plaquette) represents a nonlinear circuit element; i.e. the magnetic field energy is given by

$$
U=\left(1 / q^{2} \Lambda\right) \sum_{P}\left[1-\cos \left(q \Phi_{P}\right)\right]
$$

In total, the Lagrangian $(L=K-U)$ of the model follows from Eqs.(2),(3) and (5) to be

$$
L=(1 / 2) C \sum_{L} \dot{\phi}_{L}^{2}-\left(1 / q^{2} \Lambda\right) \sum_{P}\left[1-\cos \left(q \Phi_{P}\right)\right]
$$


together with Eq.(4). The implied Hamiltonian is

$$
H=-(1 / 2 C) \sum_{L}\left(\partial / \partial \phi_{L}\right)^{2}+\left(1 / q^{2} \Lambda\right) \sum_{P}\left[1-\cos \left(q \Phi_{P}\right)\right] .
$$

The wave functions of the theory have coordinates that live on a circle and are thereby magnetic flux quantum $(2 \pi / q)$ periodic

$$
\psi\left(\ldots, \phi_{L}+(2 \pi / q), \ldots\right)=\psi\left(\ldots, \phi_{L}, \ldots\right) .
$$

The inner product

$$
<\psi_{f}, \psi_{i}>=\oint \psi_{f}^{*}\left(\ldots, \phi_{L}, \ldots\right) \psi_{i}\left(\ldots, \phi_{L}, \ldots\right) \prod_{L}\left(q d \phi_{L} / 2 \pi\right),
$$

completes the definition (quite rigorously for a finite lattice) of the Hilbert space of states.

In the infinite lattice limit, there is one vertex per cube, three links per cube and three plaquettes per cube. On the other hand, there are only two linearly independent magnetic flux plaquette variables per cube. This arises because the magnetic field is transverse in the lattice sense to be described more fully below. Furthermore, if one starts at time zero in a subspace of states spanned by wave functions which depend only on the magetic flux coordinates

$$
\psi_{o}=\Psi\left(\ldots, \Phi_{P}, \ldots\right)
$$

then one remains in this subspace at all future times. This is a consequence of the Hamiltonian Eqs.(4) and (7). This subspace corresponds to having no charges present on the vertices. The magnetic flux coordinates here completely determine the dynamics. Let us consider this in more detail.

With link unit vectors defined as

$$
\mathbf{n}_{1}=(1,0,0), \mathbf{n}_{2}=(0,1,0), \mathbf{n}_{3}=(0,0,1)
$$

each link on the lattice is determined by a vertex and a unit vector

$$
L \mapsto(\mathbf{x}, \mathbf{n}), \quad V_{L}=a \mathbf{n} \cdot \mathbf{E}(\mathbf{x})=(i / C)\left(\partial / \partial \phi_{L}\right),
$$

where $a$ is the lattice spacing. The lattice divergence of the electric field at a vertex $\mathbf{x}$ is defined as

$$
\operatorname{div} \mathbf{E}(\mathbf{x})=(1 / a) \sum_{i=1}^{3} \mathbf{n}_{i} \cdot\left[\mathbf{E}(\mathbf{x})-\mathbf{E}\left(\mathbf{x}-a \mathbf{n}_{i}\right)\right] .
$$

On the dual lattice we have the identities

$$
\begin{gathered}
\exp \left(i q \Phi_{P}\right)=\exp \left[i q a^{2} \mathbf{n} \cdot \mathbf{B}\left(\mathbf{x}^{*}\right)\right], \\
\operatorname{div}^{*} \mathbf{B}\left(\mathbf{x}^{*}\right)=0 .
\end{gathered}
$$

The subspace of states spanned by functions of the magnetic field only, as in Eq.(10), are precisely those which obey the Gauss law with no charges at vertices

$$
\operatorname{div} \mathbf{E}(\mathbf{x}) \psi_{o}=0
$$

Now suppose that we place charges on a subset of vertices $\left\{\mathbf{x}_{j}\right\}$. The charge density (in terms of the discrete $\delta$ function) is given by

$$
\begin{aligned}
\rho(\mathbf{x}) & =\left(q / a^{3}\right) \sum_{j} z_{j} \delta\left(\mathbf{x}, \mathbf{x}_{j}\right), \\
z_{j} & = \pm 1, \pm 2, \pm 3, \pm 4, \ldots .
\end{aligned}
$$

To compute the wave functions with the external charges we must solve the Gauss law equation

$$
\operatorname{div} \mathbf{E}(\mathbf{x}) \psi=\rho(\mathbf{x}) \psi .
$$


To do so, we adopt the method used by Weyl ${ }^{(18)}$ in continous space-time QED to the problem at hand, i.e., compact lattice $\mathrm{QED}^{(19)}$. We first decompose the voltage accross a link into a transverse and longitudinal part;

$$
\begin{gathered}
V_{L}=-\dot{\bar{\phi}}(\mathbf{x}, \mathbf{n})+\dot{\chi}(\mathbf{x})-\dot{\chi}(\mathbf{x}+a \mathbf{n}), \\
\exp [i q \bar{\phi}] \equiv \exp [i q \bar{\phi}(\mathbf{x}, \mathbf{n})]=\exp [i a q \mathbf{n} \cdot \overline{\mathbf{A}}(\mathbf{x})], \\
\operatorname{div} \overline{\mathbf{A}}(\mathbf{x})=0 \\
\operatorname{div} \mathbf{E}(\mathbf{x})=\left(i / a^{3}\right)[\partial / \partial \chi(\mathbf{x})] .
\end{gathered}
$$

Only the transverse link coordinates contribute to the Faraday law magnetic fux through a plaquette. Thus, Eq.(4) may be written in terms of transverse link coordinates as

$$
\exp \left(i q \Phi_{P}\right)=\prod_{L \in \partial P} \exp \left(i q \bar{\phi}_{L}\right)
$$

From Eqs.(17) and (6) we may write the Lagrangian as a "radiation" plus an "Coulomb" part

$$
\begin{gathered}
L=(1 / 2) C \sum_{L} \dot{\bar{\phi}}_{L}^{2}-\left(1 / 2 q^{2} \Lambda\right) \sum_{P}\left[1-\cos \left(q \Phi_{P}\right)\right]+L_{\text {Coulomb }}, \\
L_{\text {Coulomb }}=(1 / 2) C \sum_{\mathbf{x}} \sum_{i=1}^{3}\left[\dot{\chi}(\mathbf{x})-\dot{\chi}\left(\mathbf{x}+a \mathbf{n}_{i}\right)\right]^{2} .
\end{gathered}
$$

The Hamiltonian has a similar decomposition

$$
\begin{gathered}
H=H_{\text {radiation }}+H_{\text {Coulomb }}, \\
H_{\text {radiation }}=-(1 / 2 C) \sum_{L}\left(\partial / \partial \bar{\phi}_{L}\right)^{2}+\left(1 / q^{2} \Lambda\right) \sum_{P}\left[1-\cos \left(q \Phi_{P}\right)\right], \\
H_{\text {Coulomb }}=-(1 / 2 C) \sum_{\mathbf{x y}} G(\mathbf{x}-\mathbf{y})\left[\partial^{2} / \partial \chi(\mathbf{x}) \partial \chi(\mathbf{y})\right],
\end{gathered}
$$

where $G(\mathbf{x}-\mathbf{y})$ is the lattice Coulomb Green's function to be discussed in more detail below. Here we note that the subspace of wave functions of the form

$$
\psi=\exp \left[i q \sum_{j} z_{j} \chi\left(\mathbf{x}_{j}\right)\right] \Psi\left(\ldots, \Phi_{P}, \ldots\right)
$$

solve the Gauss law Eq.(16) in virtue of Eqs.(10),(14),(15) and (17d).

While the radiation problem in compact lattice QED is quite complicated,

$$
H_{\text {radiation }} \Psi\left(\ldots, \Phi_{P}, \ldots\right)=E \Psi\left(\ldots, \Phi_{P}, \ldots\right),
$$

radiation is decoupled from the Coulomb energy (for static charges if not for dynamical Fermions ${ }^{(14-17)}$ ). The Coulomb energy is simply

$$
\Delta E_{\text {Coulomb }}=\left(q^{2} / 2 C\right) \sum_{i j} z_{i} z_{j} G\left(\mathbf{x}_{i}-\mathbf{x}_{j}\right)
$$

In going from the Lagrangian Eq.(19b) to the Hamiltonian Eq.(20c) one must compute the lattice Coulomb Greens function

$$
-\nabla^{2} G(\mathbf{x}-\mathbf{y})=\delta(\mathbf{x}, \mathbf{y})
$$


in which the lattice Laplace operator is here defined as

$$
-\nabla^{2} f(\mathbf{x})=\sum_{i=1}^{3}\left[2 f(\mathbf{x})-f\left(\mathbf{x}+a \mathbf{n}_{i}\right)-f\left(\mathbf{x}-a \mathbf{n}_{i}\right)\right] .
$$

Eq.(24a) may be solved by Fourier transformation as

$$
G(\mathbf{x})=(1 / 2) \oint\left[\prod_{i=1}^{3}\left(d \theta_{i} / 2 \pi\right)\right] e^{i(\theta \cdot \mathbf{x} / a)} /\left[3-\sum_{j=1}^{3} \cos \left(\theta_{j}\right)\right] .
$$

It is proved in the mathematical literature on lattice random walks that

$$
|\mathbf{x}| G(\mathbf{x}) \rightarrow(a / 4 \pi) \quad(|\mathbf{x}| \rightarrow \infty)
$$

Our genral proof of the Coulomb law for compact lattice QED is thus completed. The physical motivation of the proof (which Weyl used in ordinary space-time QED) is simply as follows: If one enforces the Gauss law on the physical wave functions, then the Coulomb law will follow. That simple reasoning is sometimes obscured in the path integral formalism unless sufficient attention is paid to the lattice Helmholtz theorem.

We make no statements as to nature of the free energy for the nonlinear radiation (transverse) degrees of freedom in lattice compact QED. But the Coulomb law for the longitudinal field components is on solid ground for all temperatures. In Fig.1, we compare the continuous space-time Coulomb potential to the Coulomb law obtained on an "infinite" lattice as in Eq.(25). The convergence to the continuous space-time Coulomb law takes about four lattice lengths. On the other hand, suppose that the calculation of $G(\mathbf{x}, \mathbf{y})$ were performed using a finite lattice with $N \times N \times N$ lattice sites. At least $N \sim 1,000$ would be required to obtain adequate numerical evidence ${ }^{(20)}$ for a long range Coulomb potential. 


\section{FIGURE CAPTION}

Fig.1: The lattice Coulomb potential in the $\mathbf{n}_{1}=(1,0,0)$ direction is compared with that obtained in the continuous space-time form of the Coulomb potential energy $\left(q^{2} z_{1} z_{2} / 4 \pi r\right)$.

\section{REFERENCES}

[1] K. G. Wilson, Phys. Rev. D10, 2445 (1974).

[2] L. P. Kadanoff, Rev. Mod. Phys. 49, 267 (1977).

[3] M. Creutz, Quarks, Gluons and Lattices, Cambridge University Press, (1983).

[4] J. Kogut and L. Susskind, Phys. Rev., D9 3501 (1974).

[5] J. Frohlich and T. Spencer, Commun. Math. Phys. 83, 411 (1982) .

[6] M. Creutz, L. Jacobs and C. Rebbi, Phys. Rev. D20, 1915 (1979) .

[7] A. H. Guth, Phys. Rev. D15, 2291 (1980) .

[8] T. A. DeGrand and D. Toussaint, Phys. Rev. D22, 2478 (1980) .

[9] B. Svetitsky, S. D. Drell, H. R. Quinn and M. Weinstein, Phys. Rev. D22, 490 (1980).

[10] R. Gupta, M. A. Novotny and R. Cordery, Phys. Lett. B172, 86 (1986) .

[11] H. Kleinert and W. Miller, Phys. Rev. Lett. 56, 11 (1986).

[12] A. N. Burkitt, Nuclear Physics, B270[FS16], 575 (1986).

[13] C. B. Lang, Nuclear Physics B280[FS18], 255 (1987)

[14] J. B. Kogut and E. Dagotto, Phys. Rev. Lett. 59, 617 (1987).

[15] M. Okawa, Phys. Rev. Lett. 62,1224 (1989) .

[16] V. Azcoiti, G. Di Carlo and A. F. Grillo, Phys. Rev. Lett. 65, 2239 (1990) .

[17] S. Hashimoto, M. Kikugawa and T. Muta, Phys. Lett. B254, 449 (1991).

[18] H. Weyl, Group Theory and Quantum Mechanics, Dover Publications, New York (1950).

[19] A. M. Polyakov, Gauge Fields and Strings, Harwood Academic Publishers, New York (1987)

[20] D. E. Knuth, The Art of Computer Programming, Vol. II Addison Wesley Publications, New York. (1969). cf. after Eq. (3.53), page 46. 